Ann. Abeille, rg66, 9 (2), I65-169.

NOTE TECHNIQUE,

\title{
AMELIORATION D'UNE TECHNIQUE DE RECHERCHE DE LA CONTAMINATION DE L'ABEILLE MARQUÉE AU MOYEN D'UN RADIO-ISOTOPE
}

\author{
P. DOUAII'T \\ Station de Recherches sur l'Abeille et les Insectes sociaun, \\ 91 - Bures-sur-Yoctle
}

\section{SOMMAIRE}

L'auteur décrit une technique facilitant la disposition d'une abeille sous l'élément de comptage d'un détecteur Geiger-Müller du type cloche.

L'abeille est introduite dans un tube de cellophane d'un diamètre approprié. L'abeille, ne pouvant faire aucun mouvement garde pendant toute la durée du comptage, une position rigoureusement constante. I.es deux extrémités du tube sont bouchées par un léger tampon de coton afin de permettre à l'abeille de respirer.

$\Lambda$ la fin du comptage, l'insecte est récupéré facilement et cette manipulation peut se répéter plusieurs fois sans inconvénient. tage.

Cette méthode s'est révélée très intéressante car elle permet d'améliorer les conditions de comp-

\section{INTRODUCTION}

Il n'est pas toujours facile de disposer sous un élément de comptage un animal vivant, même de petite taille et faire conserver à celui-ci une position constante pendant toute la durée de la détection.

Un insecte étudié au laboratoire et marqué avec un radioélément est soumis la plupart du temps à un grand nombre de manipulations, certains animaux peuvent supporter ces manipulations sans grand dommage, mais ce n'est pas le cas de l'A beille. Les anesthésies qui souvent simplifient le travail se sont avérées presque toujours préjudiciables, comme l'ont signalé de nombreux auteurs.

De plus, une abeille endormie au $\mathrm{CO}_{2}$ régurgite souvent le contenu de son jabot et par le fait même rejette une certaine quantité de radioactivité absorbée avec la nourriture (DOUAUIT, I965). 
D'autre part, il est pourtant indispensable dans bien des cas de détecter la radioactivité chez un insecte vivant. Les exigences de l'expérimentation peuvent imposer une recherche quotidienne de la radioactivité d'un insecte, aussi doit-on utiliser un dispositif permettant de placer l'animal dans les meilleures conditions de comptage.

DELVERT-SALLERON (I963) a utilisé des petites boîtes en carton $2 \times 2 \mathrm{~cm}$ dont la face tournée vers le compteur était recouverte de cellophane.

L'abeille y était introduite endormie au $\mathrm{CO}_{2}$ et devait subir à chaque comptage une nouvelle anesthésie. Cet auteur a mentionné l'inconvénient que pouvait présenter cette technique.

Nous avons donc essayé d'améliorer le système de façon à ce que :

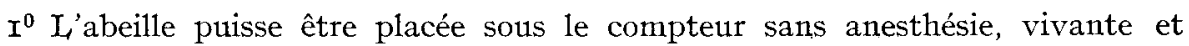
maintenue dans une position rigotıreusement constante, récupérée aisément et subir la même manipulation plusieurs jours de suite sans dommage.

$2^{0}$ L'expérimentateur puisse placer l'insecte dans les meilleures conditions de géométrie afin d'améliorer le rendement du compteur et de rendre les différents comptages plus faciles à comparer.

\section{MATÉRIEL, ET TECHNIQUE}

L'élément de comptage que nous avons utilisé est un compteur Geiger-Müller du type cloche fabriqué par le Laboratoire des Télécommunications : ${ }_{13}$ AP 7 . Nous avons travaillé à une tension constante de I 475 volts. Le mouvement propre de l'appareil est de 18 à 20 coups par minute, la cellule de comptage étant enfermée dans un château de plomb de $5 \mathrm{~cm}$ d'épaisseur.

Le mouvement propre de l'appareil (bruit de fond) a été déterminé avant chaque comptage et les résultats exprimés en coups nets, c'est-à-dire : les coups bruts moins le bruit de fond.

Nous avons choisi comme isotope le phospore 32 fourni par le C. E. A. sous forme de phosphate monosodique facilement incorporable à la nourriture. Le radio-élément a une période de désintégration de 14,3 jours. Il émet un rayonnement $\beta$ de $1,707 \mathrm{MeV}$.

Nous avons essayé plusieurs dispositifs pour maintenir l'abeille sous le tube compteur et nous avons comparé les différents résultats.

I. L'abeille était introduite vivante dans un tube de verre de $4 \mathrm{~cm}$ de long et de I $\mathrm{mm} 25$ d'épaisseur. Le tube était choisi du diamètre de l'abeille afin que celle-ci soit immobilisée parfaitement. Le tube contenant l'insecte était placé au mieux sous la cellule de comptage.

2. L'abeille était introduite vivante dans un tube de cellophane de même diamètre, lui donnant une position analogue à celle qu'elle avait dans le tube de verre.

3. L'abeille était introduite morte et non endormie pour éviter les régurgitations, dans la boîte de Delvert-Salleron fidèlement reproduite et placée sous le compteur dans les meilleures conditions d'approche et de géométrie.

4. L'abeille était déposée morte, à nu, sur un papier et disposée sous le compteur. Il est évident que pour les quatre essais il s'agissait de la même abeille, sacrifiée dans le cas des essais 3 et 4 afin d'éviter tout mouvement de sa part.

\section{Description du tube en cellophane}

Ce dispositif nous ayant donné les meilleurs résultats, comme nous allons le voir, nous donnons ici la méthode de fabrication du tube.

Il suffit de découper une bande de cellophane de $10 \mathrm{~cm}$ de long environ et de $3 \mathrm{~cm}$ de large 
maximum. Rouler cette bande sur un tube de verre de $6 \mathrm{~m} / \mathrm{m}$ de diamètre préalablement bien talqué afin de faciliter le démoulage. Coller légèrement à la jointure de la bande et retirer par un des bouts la baguette de verre.

Nous avons donc un tube de cellophane du diamètre d'une abeille, légèrement rigide tout en restant souple.

\section{ANALYSE DES RÉSULTATS}

Nous avons consigné dans le tableau des résultats les chiffres correspondant à la radioactivité d'une même abeille soumise aux différents tests.

Nous précisons tout de suite que la boîte en carton de DELVERT-SALLERON n'est pas utilisable pour faire le comptage d'une abeille vivante. L'insecte se déplaçant continuellement fait varier la distance qui le sépare du tube de comptage.

Le premier point à observer est l'immobilisation de l'abeille afin de ne pas perturber le comptage et la conservation intacte de celle-ci pour répéter autant de fois qu'on le désire la même opération.

Le tube de verre correspondant au diamètre de l'abeille convient très bien pour l'immobilisation mais est absolument inutilisable à cause de sa trop grande puissance d'arrêt des rayons bêta. En effet, comme nous le voyons, la différence de radioactivité détectée entre la boîte en carton et le tube de verre est de moins 16 p. Ioo pour ce dernier (fig. I), placé pourtant au maximum d'approche de la cellule de comptage.

FIG. 1. - Les chiffres correspondent a des coups/minute

\begin{tabular}{|c|c|c|c|c|c|c|}
\hline $\begin{array}{l}\text { Tube } \\
\text { verre }\end{array}$ & & $\begin{array}{c}\text { Tube } \\
\text { cellophane }\end{array}$ & Insecte nu & $\begin{array}{c}\text { Différ. entre } \\
\text { insecte nu } \\
\text { et tube celloph. }\end{array}$ & & $\begin{array}{l}\text { Boite } \\
\text { en carton }\end{array}$ \\
\hline $\begin{array}{r}96 \\
399 \\
655 \\
655 \\
541 \\
586 \\
548 \\
751 \\
555 \\
449 \\
204 \\
529 \\
221\end{array}$ & $\begin{array}{c}\text { Pourcentage } \\
\text { moyen } \\
\text { d'absorption } \\
\text { du tube de } \\
\text { verre par } \\
\text { rapport à la } \\
\text { boite en } \\
\text { carton : } 16 \%\end{array}$ & $\begin{array}{l}1311 \\
4958 \\
7613 \\
7613 \\
5305 \\
5 \quad 717 \\
7096 \\
8636 \\
7000 \\
4964 \\
2329 \\
6069 \\
3023\end{array}$ & $\begin{array}{l}1343 \\
5 \quad 354 \\
7674 \\
7674 \\
5576 \\
6317 \\
7100 \\
9000 \\
7250 \\
4989 \\
2340 \\
6104 \\
3217\end{array}$ & $\begin{array}{r}32 \\
396 \\
61 \\
61 \\
271 \\
600 \\
4 \\
36^{\prime} \\
250 \\
25 \\
11 \\
35 \\
19 '\end{array}$ & $\begin{array}{c}\text { Pourcentage } \\
\text { moyen } \\
\text { d'absorption } \\
\text { du tube de } \\
\text { cellophane : } \\
3,6 \% .\end{array}$ & $\begin{array}{r}623 \\
2471 \\
3821 \\
3821 \\
2676 \\
2842 \\
3628 \\
4667 \\
3232 \\
2623 \\
1297 \\
3149 \\
1728\end{array}$ \\
\hline
\end{tabular}

DELVERT-SALLERON ayant déjà constaté que l'absorption du rayonnement par le papier cellophane était parfaitement négligeable, nous avons pensé qu'en moulant cette matière sur un tube de verre correspondant dans son diamètre extérieur à celui d'une abeille, nous aurions cette fois un tube de cellophane qui peutêtre pourrait correspondre à nos besoins. 
En effet, le tube préparé par la méthode indiquée plus haut permet, avec une introduction aisée de l'abeille, de bloquer celle-ci entre deux légers tampons de coton cardé. L'abeille immobilisée totalement et pouvant respirer facilement est disposée sous le tube compteur à une distance que nous n'avions jamais pu atteindre jusqu'ici. De plus 1'abeille peut être orientée différemment et ce détail semble intéressant dans certains cas.

I'absorption du rayonnement par la cellophane est de $2,5 \mathrm{p}$. Ioo en moyenne par rapport à l'insecte compté nu, mort et simplement posé sur une feuille de papier et à une distance égale de la cellule, comme pour le précédent essai.

Nous avons étudié l'influence de la disposition de l'abeille sous la cellule de comptage et pour cela nous avons procédé de deux manières :

Un premier comptage était effectué, la face dorsale de l'insecte étant tournée vers le détecteur. Au deuxième comptage, la position était inversée. Nous avons comparé les deux méthodes et nous donnons (fig. 2) les chiffres correspondant à chacune d'elles avec la différence de coups par minute.

Fra. 2. - Les chiffres correspondent à des coups/minule

\begin{tabular}{|c|c|c|c|}
\hline $\begin{array}{c}\text { Face dorsale } \\
\text { vers le compteur }\end{array}$ & $\begin{array}{l}\text { Face ventrale } \\
\text { vers le compteur }\end{array}$ & $\begin{array}{l}\text { Différence entre } \\
\text { les } 2 \text { comptages }\end{array}$ & \\
\hline $\begin{array}{l}1210 \\
3480 \\
2818 \\
2976 \\
2499^{\prime} \\
1229 \\
4 k 992 \\
3491 \\
2244 \\
419 \prime \\
4391 \\
2215 \\
2622\end{array}$ & $\begin{array}{l}1209 \\
3629 \\
2992 \\
3302 \\
2472 \\
1948 \\
5294 \\
388 t \\
2236 \\
4386 \\
4730 \\
2264 \\
2803\end{array}$ & $\begin{array}{rr}- & 1 \\
+ & 219 \\
+ & 174 \\
+ & 326 \\
-\quad 22 \\
+\quad 19 \\
+ & 302 \\
+ & 393 \\
-\quad 8 \\
+ & 192 \\
+ & 339 \\
+\quad 49 \\
+\quad 181\end{array}$ & $\begin{array}{l}\text { Pourcentage } \\
\text { moyen en } \\
\text { faveur du } \\
\text { comptage : } \\
\text { face ventrale : } \\
+4 \%\end{array}$ \\
\hline
\end{tabular}

Comme nous le remarquons, il y a une légère différence entre les deux comptages et il semble préférable de " compter " l'abeille par la face ventrale. Il est possible que cette différence soit attribuable au contenu de l'ampoule rectale, cet organe se trouvant plus rapproché de la face ventrale que de la face dorsale.

Enfin, nous tenons à signaler, que les abeilles manipulées quotidiennement pour être introduites dans le tube en cellophane ne subissent aucun dommage. Nous avons fait une étude de la survie sur deux lots de ro abeilles qui étaient introduites chaque jour dans les tubes et comparées à un même nombre de témoins non manipulés.

Pendant les ro jours correspondant à la durée de l'expérience nous n'avons enregistré qu'une mortalité et par ailleurs, nous n'avons pas remarqué de modifications dans le comportement des abeilles soumises au test. 


\section{CONCLUSION}

Les différents dispositifs que nous avions utilisés jusqu'à la fabrication des tubes en cellophane ne nous avaient jamais apporté la qualité de comptage que nous recherchions. Les tubes sont d'une fabrication simple et peu coûteuse, étant jetés après usage, ils éliminent tout travail de décontamination du matériel, toujours fastidieux.

Ils permettent de réaliser des conditions de géométrie très satisfaisantes et ainsi d'améliorer la qualité du comptage, sa fidélité et les possibilités de comparaison.

Notons que la méthode n'est pas seulement applicable à l'abeille mais doit pouvoir être utilisée dans presque tous les cas où l'on désire étudier la radioactivité d'un insecte vivant.

$$
\text { Rę̧u pour publication en mars } 1966 .
$$

\section{RÉFÉRENCES BIBLIOGRAPHIQUES}

Delvert-Salleroy F., I963. Étude au moyen de radio-isotopes des échanges de nourriture entre reines, mâles et ouvrières d'Apis mellifica L. Ann. Abeille, 6, 201-227.

Douacit Ph., 1966. Étude de la contamination externe de l'Abeille et de son milieu par un radio-isotope introduit dans la nourriture. Ann. Abeille, 9, 37-45. 